\title{
BMJ Open Effects of esmolol on QTc interval changes during tracheal intubation: a systematic review
}

\author{
Venkatesan Thiruvenkatarajan, ${ }^{\oplus 1}$ Jenn Yuan Lee, ${ }^{2}$ Manesha Sembu, ${ }^{3}$ \\ Richard Watts, ${ }^{1}$ Roelof Markus Van Wijk ${ }^{1}$
}

To cite: Thiruvenkatarajan V, Lee JY, Sembu M, et al. Effects of esmolol on QTC interval changes during tracheal intubation: a systematic review. BMJ Open 2019;9:e028111. doi:10.1136/ bmjopen-2018-028111

- Prepublication history and additional material for this paper are available online. To view these files, please visit the journal online (http://dx.doi. org/10.1136/bmjopen-2018028111).

Received 24 November 2018 Revised 18 February 2019 Accepted 13 March 2019

\section{Check for updates}

C Author(s) (or their employer(s)) 2019. Re-use permitted under CC BY-NC. No commercial re-use. See rights and permissions. Published by BMJ.

${ }^{1}$ Anaesthesia, The Queen Elizabeth Hospital, Adelaide, South Australia, Australia

${ }^{2}$ The University of Adelaide, Adelaide, South Australia, Australia

${ }^{3}$ University of New South Wales, Sydney, New South Wales, Australia

\section{Correspondence to}

Dr Venkatesan

Thiruvenkatarajan;

Venkatesan.Thiruvenkatarajan@ sa.gov.au

\begin{abstract}
Introduction and aims Esmolol is an ultra-shortacting $\beta_{1}$ antagonist that has been shown to attenuate the corrected QT (QTc) interval prolongation associated with laryngoscopy and endotracheal intubation (LTI). Prolongation of the QTc interval can precipitate arrhythmias, the most serious of which is torsades de pointes. The aim of this systematic review was to compare esmolol and placebo on QTc changes occurring during LTI.
\end{abstract}

Materials and methods PubMed, EMBASE, Cochrane Registry of Clinical Trials and CINAHL databases (up to August 2018) were screened for randomised controlled trials comparing esmolol and placebo on QTc changes during LTI in cardiac and non-cardiac surgeries. The primary outcome was QTC changes during LTI and secondary outcome was related to adverse effects from esmolol such as bradycardia and hypotension.

Results Seven trials were identified involving 320 patients, 160 patients receiving esmolol or placebo apiece. A shortening of the QTC post-LTI was evident in the esmolol group compared with the placebo in four studies. Compared with the baseline, the QTc was reduced post-LTI in the esmolol group. In the placebo group, the QTC was prolonged compared with the baseline post LTI. Nonetheless, esmolol did not prevent QTC prolongation in the remaining three studies, and much of this was attributed to employing QTC prolonging agents for premedication and anaesthetic induction. No significant adverse events were noted.

Conclusion Compared with placebo, esmolol reduced the LTI-induced QTC prolongation when current non-QTC prolonging agents were chosen for tracheal intubation. Future studies should explore whether transmural dispersion (a marker of torsadogenicity) is also affected during LTI by analysing parameters such as the Tp-e interval (interval between the peak to the end of the T-wave) and Tp-e/QTc (rate corrected Tp-e interval). Trial registration number CRD42018090282.

\section{INTRODUCTION}

Prolongation of the corrected QT (QTc) interval of the electrocardiogram (QT interval corrected for heart rate) can occur throughout anaesthetic induction and tracheal intubation, maintenance and emergence during a general anaesthetic. ${ }^{1}$ Airway

\section{Strengths and limitations of this study}

This is the first systematic review to explore the effect of an adjuvant medication in mitigating the corrected QT response secondary to laryngoscopy and tracheal intubation.

- The Preferred Reporting Items for Systematic Reviews and Meta-analyses guidelines were strictly adhered to in conducting this review.

- Studies were included irrespective of the language and trials were spread across a diverse subset; for example, American Society of Anaesthesiologists I-II to cardiac surgeries.

- Medication regimen chosen by the older studies are no longer applicable in contemporary practice, thus contributed to heterogeneity.

manoeuvres such as laryngoscopy and tracheal intubation (LTI) are associated with an intense sympathetical surge and have been identified as critical periods of QTc interval disturbances precipitating arrhythmias. ${ }^{1} 2$ Torsades de pointes (TdP) has been described during LTI. ${ }^{3}$ The use of a supraglottic airway device has been shown to produce less QTc perturbations compared with LTI. ${ }^{4}$ While this may not be a significant concern in otherwise healthy patients, it can lead to significant morbidity in specific high-risk patient population. This group includes those with coronary artery disease,$^{5-7}$ hypertension, ${ }^{89}$ and patients undergoing coronary artery bypass grafting (CABG) procedures. ${ }^{10}$ Hypertension could exacerbate the sympathetical response during LTI predisposing to QTc changes apart from haemodynamic response. ${ }^{8}$ QTc interval prolongation has been identified as a risk factor for cardiovascular events in hypertensive as well as diabetic patients. ${ }^{11} 12$ Perioperative QTc prolongation predisposes to complications such as polymorphic ventricular tachycardia, myocardial ischaemia and sudden cardiac death. ${ }^{2}$ Nonetheless, this phenomenon is probably less appreciated in clinical practice. 
Various agents have been used during induction of general anaesthesia to attenuate LTI-induced QTc prolongation such as beta blockers, ${ }^{7-9}$ intravenous lignocaine ${ }^{13}$ and opioids (fentanyl and remifentanil). ${ }^{14-16}$ Esmolol, a selective $\beta_{1}$ antagonist, is among one of the most studied drugs, due to its ability to dampen the sympathetic tone, which is one of the underlying mechanisms of QTc prolongation.

The primary objective of this systematic review was to evaluate the effect of esmolol compared with control, in mitigating the LTI-induced QTc interval prolongation in adult patients aged 18 years and above who were undergoing elective surgery.

The secondary objective was to define any adverse effects associated with esmolol administration during anaesthetic induction and LTI.

\section{METHODS}

The review was conducted in compliance with the Preferred Reporting Items for Systematic Reviews and Meta-Analyses (PRISMA) guidelines and the protocol was registered on PROSPERO.

\section{Search strategy}

The relevant studies were identified through PubMed, EMBASE and Cochrane Central Registry of Clinical Trials and CINAHL databases, and were subsequently recruited by applying our inclusion criteria. The initial literature search strategy on PubMed included appropriate use of medical subject headings (MeSH) terms, adequate descriptors and Boolean operators and was performed as follows: ([esmolol] and [anaesthesia or general anaesthesia or induction of anaesthesia] and [QT interval or QTc interval]). Details of the search strategy is given in online supplementary appendix 1. Complementary search strategies were used for other databases according to their particulars. A manual search was performed on the articles that were cross-referenced in the selected studies. No language restriction was applied.

\section{Study selection}

Two authors (VT and JYL) independently assessed abstracts and titles of all the studies that were a potential inclusion based on the search strategy. The non-English abstracts were translated with google translate, and if found eligible or ambiguous, full translation of the manuscript was undertaken. The full texts of all eligible studies were independently screened by two investigators (VT and JYL), any discrepancies were resolved by discussion and consensus. The PRISMA flow chart for selection of studies is shown in figure 1.

\section{Eligibility criteria}

Studies were included based on the following criteria: (1) randomised trials in adult patients comparing fixed or various doses of esmolol with an active placebo control during induction of anaesthesia and (2) reporting various

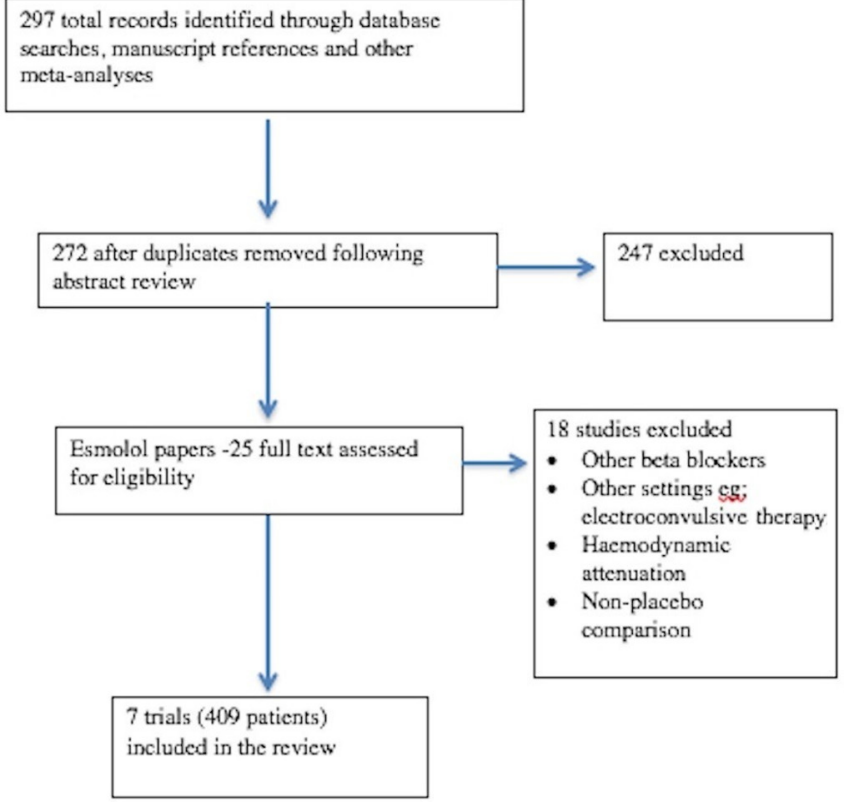

Figure 1 The Preferred Reporting Items for Systematic Reviews and Meta Analyses flowchart reflecting the literature search process.

parameters of the QT interval such as QTc, QTd ([QT dispersion], difference between maximum and minimum QT), QTcd (QTd corrected for heart rate), Tp-e interval (interval between the peak to the end of the T-wave) and Tp-e/QTc (rate corrected Tp-e interval). It was considered essential that the studies reported baseline values of the QTc parameters prior to esmolol administration as well as at least two values reported within 5 min of endotracheal intubation. Non-controlled studies and studies where airway management was carried out without a single lumen endotracheal tube (eg, supraglottic airway) were excluded.

\section{Data extraction and definition of outcome parameters}

A data extraction sheet (template attached as online supplementary appendix 2) was created that contained information on the following: author, year and journal, American Society of Anaesthesiologists (ASA) physical status, type of surgery, cohort type in terms of co-morbidities, nature of premedication, induction agents, dose and timing of esmolol, QT parameters assessed, formula used for QTc correction, timing of assessment of QT interval post intubation, type of control (placebo or no treatment), any adverse events recorded such as severe bradycardia or other arrhythmias or allergic reactions. Also, conflicts of interest and source of funding were also extracted. Two authors (VT and DL) independently extracted the data from the included trials and discrepancies were resolved by discussion and consensus. Data were originally extracted from text or tables and from figures or graphs if not available in tables.

Our primary outcome included all the QT parameters captured at any time frame during the first 5 min after LTI. The difference in outcome between the esmolol and 
the control group within the 5 min post-LTI were assessed. Secondary outcomes were significant adverse events such as bradycardia, hypotension and arrhythmias.

\section{Risk of bias assessment}

The risk of bias was assessed using the Cochrane Collaboration's risk of bias tool and the risk of bias was summarised. Three authors independently assessed the risk of bias for each study, and disagreements resolved by consensus.

\section{Patient and public involvement}

There were involvement of any patients or the public in the study.

\section{RESULTS}

The search and recruitment of papers were performed up to August 2018. The search strategy generated a total of 297 citations. The full texts of 25 articles were screened for further relevance after excluding 272 articles based on consideration of the title and abstract and duplicates. We included seven randomised controlled trials (RCTs) with a total of 409 participants. Of the included trials, six were in English and one in Chinese. Figure 1 depicts the flow diagram of the studies, screened, identified and retained as per the PRISMA guidelines.

The details of the included studies are shown in table 1. Of the seven studies included, four compared esmolol versus placebo, ${ }^{7-10} 17$ whereas in three studies esmolol was compared with placebo as well as with an alternative therapeutic agent. ${ }^{18-20}$ We analysed data from 409 patients of whom 160 each had either received esmolol or placebo (320 in total). The remaining 89 had received alternative therapeutic agents mostly opiates or lignocaine. The average trial size was 58 patients (range 40 to 80 ).

Only one study reported the data on variance of the QT parameter. Further, the studies varied in terms of the population that was assessed, adjuvant drugs used during the intubation process, mode of administration (some studies used just a bolus while others used bolus followed by an infusion), timings of assessing the QT parameters and the formulas used in analysing. As a result of these clinical and methodological heterogeneity, a qualitative narrative synthesis of the findings are presented.

\section{Study population}

Four studies had patients with an ASA status I-II undergoing various elective surgeries ${ }^{17-20}$ two had patients with documented coronary artery disease ${ }^{7}$ and hypertension ${ }^{9}$ respectively and one study was conducted in patients undergoing $\mathrm{CABG}{ }^{10}$

\section{Premedication regime/induction agents/anaesthetic agents}

Premedication administration was described in five studies. While midazolam was used in two studies (one intramuscular and other unspecified route), ${ }^{10} 18$ intramuscular oxycodone and atropine was used in three studies. ${ }^{17} 1920$ Thiopentone sodium and suxamethonium were used as induction agents in three studies, ${ }^{17} 1920$ propofol and vecuronium in three studies, ${ }^{79} 18$ etomidate and vecuronium in one study. ${ }^{10}$ Opiates were used during induction in three studies, ${ }^{7910}$ whereas four studies did not use opioids at induction. ${ }^{17-20}$

\section{Administration of study drug}

Several esmolol regimens were tested including intravenous bolus, infusion or combination of both. Five

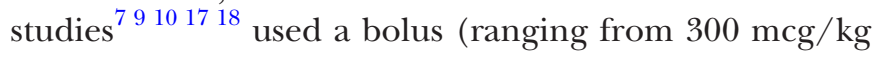
to $1000 \mathrm{mcg} / \mathrm{kg}$ ) followed by infusion (ranging from 100 $\mathrm{mcg} / \mathrm{kg} / \mathrm{min}$ to $250 \mathrm{mcg} / \mathrm{kg} / \mathrm{min}$ ). Of the two remaining studies, one study used a bolus alone at $2 \mathrm{mg} / \mathrm{kg},{ }^{20}$ and the remaining study used two different combination of boluses, $2 \mathrm{mg} / \mathrm{kg}$ and $3 \mathrm{mg} / \mathrm{kg} .{ }^{19}$ Esmolol was commenced from $1 \mathrm{~min}$ up to $5 \mathrm{~min}$ prior to administration of induction agents. Most of the infusions were ceased $4 \mathrm{~min}$ post LTI.

\section{QT interval assessment}

The QTc analysis was carried out at multiple time frames post intubation from $30 \mathrm{~s}$ until $10 \mathrm{~min}$. All the studies assessed corrected QTc, and one study assessed QTcd as well. ${ }^{9}$ Bazzett's formula was used for rate correction in five studies, whereas the remaining studies used Hodges ${ }^{10}$ and Fredericia's ${ }^{7}$ formulas, respectively. A QTc of $440 \mathrm{~ms}$ was considered normal regardless of the gender of the patients.

\section{Outcomes}

The mean baseline QTc was $424 \mathrm{~ms}$ in the esmolol group and $420 \mathrm{~ms}$ in the control group. Four studies ${ }^{79} 1018$ (recruiting patients with known coronary artery disease, known hypertensives, undergoing CABG and ASA status I-II) have shown consistent results in terms of esmolol's effect on the QTc interval. Prolongation of QTc was observed in the control group post LTI. The groups treated with esmolol had a reduced QTc interval compared with the controls. Further, compared with the baseline values, elevation of the QTc interval was not observed at any time post LTI in the esmolol group. Nonetheless, compared with the baseline values, QTc was prolonged post LTI in the control group. A similar pattern post LTI was noted for QTcd as well in patients treated with esmolol compared with controls. ${ }^{9}$ The mean QTcd was $19 \mathrm{~ms}$ in the esmolol group and $24 \mathrm{~ms}$ in the control group, and the maximun QTcd noted in the control group after intubation was 35 ms.

Esmolol did not prevent the LTI QTc prolongation in three studies that used thiopentone and suxamethonium (without opioids) for intubation following intramuscular atropine premedication. ${ }^{17} 1920$ The maximum QTc observed was $450 \mathrm{~ms}$ in the trials that avoided thiopentone and suxamethonium, whereas it was $490 \mathrm{~ms}$ in trials that employed those agents. The summary of findings is presented in table 2.

\section{Risk of bias}

The potential sources of bias have been outlined in figure 2 and an overall estimate of the risk of bias has 


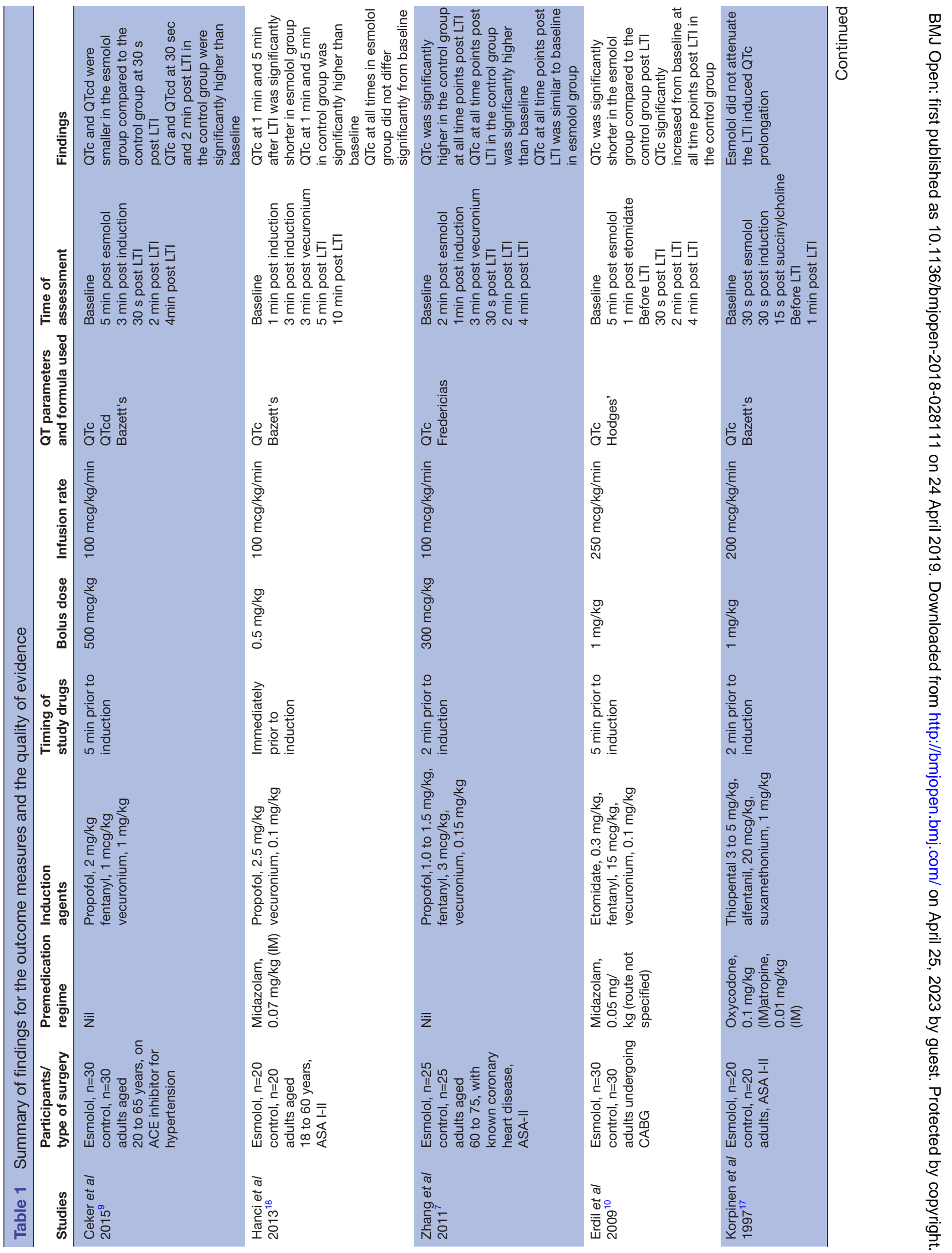




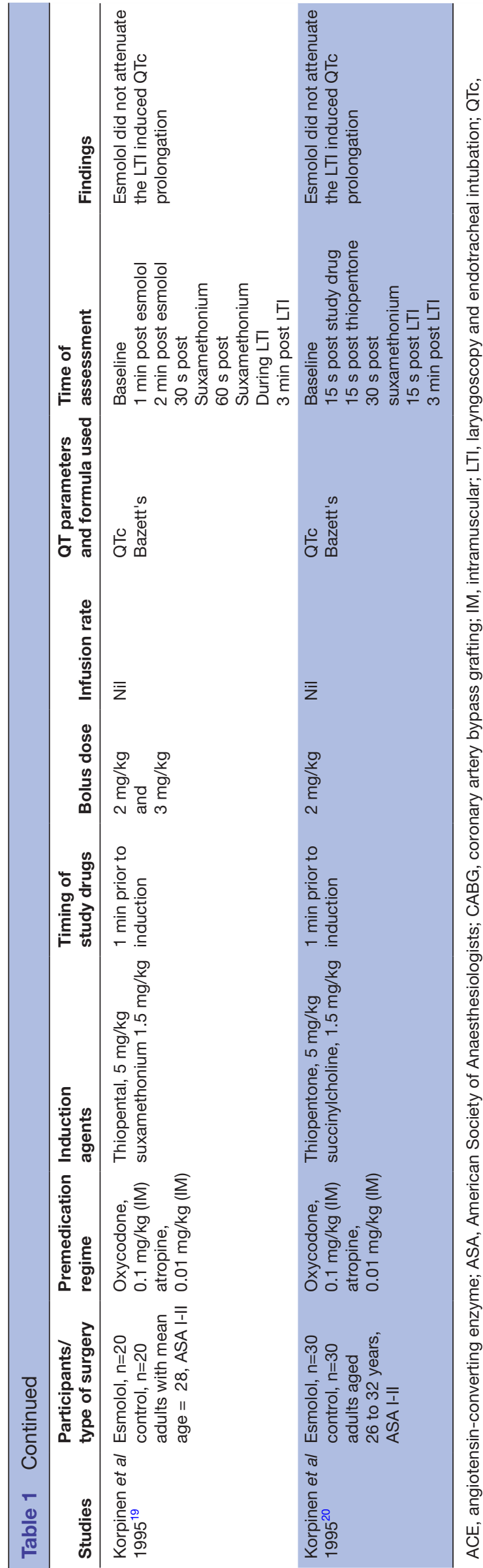

been created for each study. Five of the seven studies were of sufficiently good quality with a low risk of bias.

\section{Adverse effects}

Adverse events were reported in two trials, one involving hypertensive patients and the other analysing patients undergoing CABG. ${ }^{9}{ }^{10}$ Of the combined 120 patients in these studies (60 esmolol and 60 control), eleven patients in the control group developed less severe ventricular arrhythmias as compared with two in the esmolol group. The difference was reported as significant in one study. No other haemodynamic adverse events were reported in any of the trials.

\section{Source of funding for the included studies}

Five studies did not report any conflict of interest or source of funding. Two studies by the same sources (done in 1995) reported support from Paivikki and Sakari Sohlberg foundation. ${ }^{19} 20$ This foundation supports the wellbeing of children and seniors in Finland.

\section{DISCUSSION}

The main findings from this review are that compared with placebo, esmolol administration during anaesthetic induction attenuated the QTc prolongation post LTI. This was evident only in studies that used anaesthetic inducing agents that had no effect on the QTc interval. ${ }^{79} 1018$ Meta-analyses was precluded by the lack of data on variability and the clinical and methodological heterogeneity of the included studies.

Our review had three trials done in the 1990's by a single group ${ }^{171920}$ and four studies done in the last decade since 2009. ${ }^{7} 1018$ Interestingly, the three older studies had used agents that could prolong the QTc interval, such as atropine and suxamethonium (without opioids) prior to intubation. Unsurprisingly, esmolol did not reduce the LTI-induced QTc prolongation in those trials. However, the later studies that used non-QTc disturbing induction medications showed a consistent protective effect on LTI-induced QTc response. Esmolol was noted to blunt the QTc prolongation compared with the control group and no prolongation was noticed compared with the baseline in the esmolol group unlike the control group.

There are multiple theories as to how esmolol is able to achieve its effects on the QTc interval. Esmolol, being a $\beta_{1}$-adrenergic antagonist is able to directly mitigate the heightened sympathetic tone caused by LTI. Coupled with an anti-ischaemic effect, the antisympathetical effect helps in reducing the QTc prolongation. ${ }^{10}$ Similar effects have been shown for other beta blockers such as landiolol. ${ }^{21}$

The favourable pharmacokinetic profile (direct alpha antagonism, ultra-short acting, rapid onset and offset) makes it an ideal agent in this regard. Also, it can be employed as an infusion post intubation in vulnerable patients or those who present with a baseline acquired QTc prolongation if their perioperative pathway is likely 
Table 2 Summary of findings for the outcome measures and the quality of evidence (GRADE, Grading of Recommendations, Assessment, Development and Evaluation)

\begin{tabular}{|c|c|c|c|}
\hline Outcome & Effect & $\begin{array}{l}\text { Number of participants } \\
\text { (studies) }\end{array}$ & $\begin{array}{l}\text { Quality of the evidence } \\
\text { (GRADE) }\end{array}$ \\
\hline \multicolumn{4}{|c|}{$\begin{array}{l}\text { Patient or population: surgical patients with the need for general anaesthesia with an endotracheal tube } \\
\text { Settings: induction of general anaesthesia and endotracheal intubation. } \\
\text { Intervention: esmolol administration prior to endotracheal intubation. }\end{array}$} \\
\hline $\begin{array}{l}\text { QTC post LTI: esmolol versus } \\
\text { control }\end{array}$ & $\begin{array}{l}\text { four of the seven studies } \\
\text { showed a consistent reduction } \\
\text { in the esmolol group }\end{array}$ & 409 (7) & $\begin{array}{l}\text { Moderate certainty } \\
\bigoplus \oplus \bigoplus O \text { (due to risk of bias } \\
\text { from two studies) }\end{array}$ \\
\hline $\begin{array}{l}\text { QTC post LTI: baseline } \\
\text { versus post LTI }\end{array}$ & $\begin{array}{l}\text { four studies showed a } \\
\text { reduction within the esmolol } \\
\text { group }\end{array}$ & 409 (7) & $\begin{array}{l}\text { Moderate certainty } \\
\bigoplus \bigoplus \bigoplus O \text { (due to risk of bias } \\
\text { from two studies) }\end{array}$ \\
\hline
\end{tabular}

\section{Comparison:}

1. QTC interval parameters post endotracheal intubation between the group receiving esmolol and the control group receiving placebo

2. Comparison of the QTC interval parameters between the baseline and post endotracheal intubation within the esmolol and the control group

\begin{tabular}{|c|c|c|c|}
\hline $\begin{array}{l}\text { QTcd post LTI: esmolol versus } \\
\text { control }\end{array}$ & $\begin{array}{l}\text { One study showed a reduction } \\
\text { in the esmolol group }\end{array}$ & $60(1)$ & $\begin{array}{l}\text { Very low certainty } \\
\oplus 000 \text { (due to } \\
\text { imprecision, }<400 \text { participants) }\end{array}$ \\
\hline $\begin{array}{l}\text { QTcd post LTI: Baseline } \\
\text { versus post LTI }\end{array}$ & $\begin{array}{l}\text { One study showed a reduction } \\
\text { within the esmolol group }\end{array}$ & 60 (1) & $\begin{array}{l}\text { Very low certainty } \\
\oplus 000 \text { (due to } \\
\text { imprecision, }<400 \text { participants) }\end{array}$ \\
\hline
\end{tabular}

LTI, laryngoscopy and endotracheal intubation; QTc, corrected QT; QTcd, QTd corrected for heart rate (QTd, QT dispersion).

to be vulnerable to QTc triggering insults. Indeed, beta blockers are the first line therapeutical options for congenital long QT syndrome (LQTS). ${ }^{2}$ Although some sources have used opioids alone and shown a favourable QTc response post LTI, ${ }^{14-16}$ it is conceivable that

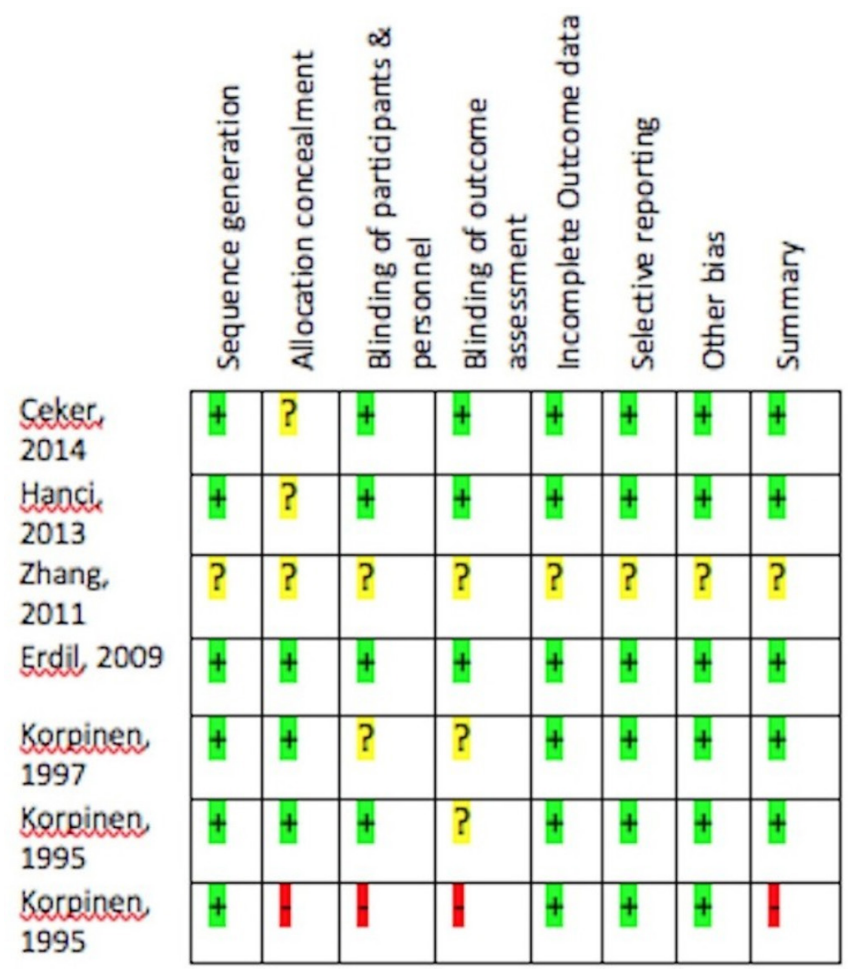

Figure 2 Risk of bias assessment of included studies. esmolol may have more profound sympatholytical effect compared with opioids. Because it is a standard practice to use opioids at induction, a synergistical effect on the QTc interval could be anticipated with co-administration of esmolol. The aforementioned properties of esmolol makes it a unique agent during anaesthetic induction and LTI offering protection against both QTc and haemodynamic response. Yet, esmolol should be cautiously used during anaesthesia induction and tracheal intubation in view of its potent haemodynamic effects.

Perioperative QTc interval burden has been a subject of interest recently. Acquired LQTS is grossly underrecognised in the perioperative period, and it warrants caution when encountered. ${ }^{2}$ The perioperative management of LQTS is based on low level of evidence, and no consensus guidelines exist. ${ }^{2}$ Minor QTc interval prolongation (an average of $23 \mathrm{~ms}$ ) up to 48 hours postoperatively was noted in $80 \%$ of patients in a cohort of 469 patients undergoing non-cardiac procedures. A QTc over $440 \mathrm{~ms}$ was noted in $51 \%$ of them, $4 \%$ had marked QTc prolongation (over $500 \mathrm{~ms}$ ), and one patient developed TdP. ${ }^{22}$ A combined data on 1600 patients across three studies has shown that preexisting QTc prolongation is an independent risk factor for major postoperative cardiovascular events. ${ }^{23-25}$ For every $10 \mathrm{~ms}$ prolongation of the QTc interval above $436 \mathrm{~ms}$, a $13 \%$ increase in adverse events were observed. ${ }^{23}$

In the non-operative environment, the risk of TdP increases either when the QTC interval exceeds 500 $\mathrm{ms}$ or with a $20 \mathrm{~ms}$ increase from baseline. ${ }^{26}{ }^{27}$ In the 
perioperative setting, an increase of over $100 \mathrm{~ms}$ from baseline was noted as a risk factor for TdP in a recent systematic review analysing the reported cases of perioperative TdP. ${ }^{3}$ The same review found that during the TdP event, the mean QTc was $575 \mathrm{~ms}$. It has been proven that prolongation of the QTc interval alone is not a marker for TdP development. Unless the transmural dispersion of the myocardium is also distorted, the risk of TdP is less. ${ }^{27}$ It is reflected by intervals other than QTc such as the Tp-e interval, Tp-e/QTc ratio. Although it is known that the transmural dispersion of repolarisation is affected by anaesthetic and other perioperative agents, it is unclear whether airway manipulations elicit similar effects. Nevertheless, vigilance is required in the susceptible whenever the perioperative milieu is likely to be exposed to QTc modifying factors.

Our review could not find major adverse events or serious haemodynamic compromise with esmolol administration during anaesthetic induction and tracheal intubation. The number of subjects in our review was probably low for this outcome. A large review assessing the safety of prolonged esmolol infusion following a bolus failed to show significant bradycardia as an adverse event. ${ }^{28}$ Likewise, another meta-analysis on the safety of esmolol demonstrated no significant hypotension or bradycardia in non-cardiac surgery. ${ }^{29}$ The authors concluded that the ultra-short acting nature of esmolol only causes reversible episode of hypotension and bradycardia, and the concerns of a negative inotropic effect are minimal. It is plausible that employing esmolol for shorter duration during LTI may not produce significant haemodynamic changes since it is likely to counter balance the sympathetical surge induced hypertension and tachycardia. Preparation of an esmolol infusion may insert a layer of complexity during anaesthetic induction, yet, the best clinical management should not be influenced by comfort, as echoed by a meta-analysis evaluating the effects of esmolol in attenuating the haemodynamic response during LTI. $^{30}$ The meta-analysis on 38 trials including 2009 patients found that esmolol was very effective in attenuating the haemodynamic response to LTI. ${ }^{30}$ Ultra-short acting beta blockers have a wide range of application in the perioperative and critical care setting including cardiac surgery, acute myocardial infarction, critically ill septic patients, improving oxygenation in extracorporeal membrane oxygenation and perioperative cardioprotection for non-cardiac surgery. ${ }^{31}$

This review had few shortcomings. The inconsistency noted in the choice of inducing agents attributed to earlier trials is a limitation. Varying dose range was described, and a dose- seeking pilot study was attempted in only three of the trials. Adverse effects were poorly reported.

An extensive literature search without language restriction is a major strength of our review. Our review offers scope to design future trials. Hodges' formula may be appropriate to evaluate the QTc parameters during LTI as it is less likely to be influenced by heart rate changes. ${ }^{7}$

\section{CONCLUSION}

The review on seven RCTs presents the evidence that esmolol can prevent LTI0induced QTc prolongation compared with placebo, in healthy patients as well as in those at risk. This effect was appreciated when non-QT prolonging anaesthetic medications were chosen to facilitate induction and LTI. No major adverse events were reported. A loading dose of $500 \mathrm{mcg} / \mathrm{kg}$ followed by an infusion of 100 to $200 \mathrm{mcg} / \mathrm{kg} / \mathrm{min}$ continuing for at least 5 to $7 \mathrm{~min}$ post LTI seems logical. Esmolol is uniquely poised to attenuate both the QTc response as well as the haemodynamical response secondary to LTI. These findings may have clinical implications in patients at risk of developing QTc interval prolongation perioperatively. Future studies should explore whether transmural dispersion is also affected during LTI by analysing parameters such as Tp-e and Tp-e/QTc apart from identifying the safest dose regime.

Contributors VT conceived the review, designed the protocol and wrote the manuscript. VT and JYL were involved in the literature search, data extraction and study quality assessment. MS assisted in data analysis, study quality and PRISMA checklist. RW conceived the idea, assisted in the discussion. RVW assisted with clinical suggestions, data analysis and discussion. All the authors reviewed and approved the final version.

Funding The authors have not declared a specific grant for this research from any funding agency in the public, commercial or not-for-profit sectors.

Competing interests None declared.

Patient consent for publication Not required.

Provenance and peer review Not commissioned; externally peer reviewed.

Data sharing statement No additional data are available.

Open access This is an open access article distributed in accordance with the Creative Commons Attribution Non Commercial (CC BY-NC 4.0) license, which permits others to distribute, remix, adapt, build upon this work non-commercially, and license their derivative works on different terms, provided the original work is properly cited, appropriate credit is given, any changes made indicated, and the use is non-commercial. See: http://creativecommons.org/licenses/by-nc/4.0/.

\section{REFERENCES}

1. Fazio G, Vernuccio F, Grutta G, et al. Drugs to be avoided in patients with long QT syndrome: Focus on the anaesthesiological management. World J Cardiol 2013;5:87-93.

2. O'Hare M, Maldonado Y, Munro J, et al. Perioperative management of patients with congenital or acquired disorders of the QT interval. Br J Anaesth 2018;120:629-44.

3. Johnston J, Pal S, Nagele P. Perioperative torsade de pointes: a systematic review of published case reports. Anesth Analg 2013;117:559-64.

4. Byon HJ, Song J, Shinn HK, et al. A comparison of QTc intervals following laryngoscopic intubation and i-gel insertion during propofol-sevoflurane anesthesia. Minerva Anestesiol 2016;82:950-6.

5. Ay B, Fak AS, Toprak A, et al. QT dispersion increases during intubation in patients with coronary artery disease. $J$ Electrocardiol 2003;36:99-104.

6. Lischke V, Behne M, Doelken P, et al. Droperidol causes a dose-dependent prolongation of the QT interval. Anesth Analg 1994;79:983-6.

7. Zhang ZP, Mr G, Fang NN, et al. The effect of esmolol on the QTC interval during tracheal intubation in elderly patients with coronary artery disease. Anaesthesia 2011;27:464.

8. Kim EJ, Han DW, Song MK, et al. Effect-site concentration of remifentanil for attenuating QTc interval prolongation following intubation in hypertensive female patients. Acta Anaesthesiol Scand 2015;59:1269-77.

9. Ceker Z, Takmaz SA, Baltaci B, et al. [The effect of esmolol on corrected-QT interval, corrected-QT interval dispersion changes 
seen during anesthesia induction in hypertensive patients taking an angiotensin-converting enzyme inhibitor]. Rev Bras Anestesiol 2015;65:34-40.

10. Erdil F, Demirbilek S, Begec Z, et al. The effect of esmolol on the QTc interval during induction of anaesthesia in patients with coronary artery disease. Anaesthesia 2009;64:246-50.

11. Dimopoulos S, Nicosia F, Turini D, et al. Prognostic evaluation of QTdispersion in elderly hypertensive and normotensive patients. Pacing Clin Electrophysiol 2009;32:1381-7.

12. Clemente D, Pereira T, Ribeiro S. Ventricular repolarization in diabetic patients: characterization and clinical implications. Arq Bras Cardiol 2012;99:1015-22.

13. Owczuk R, Wujtewicz MA, Sawicka W, et al. The effect of intravenous lidocaine on QT changes during tracheal intubation. Anaesthesia 2008;63:924-31.

14. Cafiero T, Di Minno RM, Di lorio C. QT interval and QT dispersion during the induction of anesthesia and tracheal intubation: a comparison of remifentanil and fentanyl. Minerva Anestesiol 2011;77:160-5

15. Kweon TD, Nam SB, Chang $\mathrm{CH}$, et al. The effect of bolus administration of remifentanil on QTc interval during induction of sevoflurane anaesthesia. Anaesthesia 2008;63:347-51.

16. Chang DJ, Kweon TD, Nam SB, et al. Effects of fentanyl pretreatment on the QTc interval during propofol induction. Anaesthesia 2008;63:1056-60.

17. Korpinen R, Simola M, Saarnivaara L. Effect of esmolol on the heart rate, arterial pressure and electrocardiographic changes during laryngomicroscopy. Acta Anaesthesiol Scand 1997;41:371-5.

18. Hancı V, Yurtlu S, Karabağ T, et al. Effects of esmolol, lidocaine and fentanyl on P wave dispersion, QT, QTc intervals and hemodynamic responses to endotracheal intubation during propofol induction: a comparative study. Braz J Anesthesiol 2013;63:235-44.

19. Korpinen R, Saarnivaara L, Siren K. QT interval of the ECG, heart rate and arterial pressure during anaesthetic induction: comparative effects of alfentanil and esmolol. Acta Anaesthesiol Scand 1995;39:809-13.

20. Korpinen R, Saarnivaara L, Siren K, et al. Modification of the haemodynamic responses to induction of anaesthesia and tracheal intubation with alfentanil, esmolol and their combination. Can $J$ Anaesth 1995;42:298-304.
21. Kaneko M, Yamaguchi S, Hamaguchi S, et al. Effects of landiolol on QT interval and QT dispersion during induction of anesthesia using computerized measurement. J Clin Anesth 2009;21:555-61.

22. Nagele P, Pal S, Brown F, et al. Postoperative QT interval prolongation in patients undergoing noncardiac surgery under general anesthesia. Anesthesiology 2012;117:321-8.

23. Biteker M, Duman D, Tekkeșin Al. Predictive value of preoperative electrocardiography for perioperative cardiovascular outcomes in patients undergoing noncardiac, nonvascular surgery. Clin Cardiol 2012;35:494-9.

24. Payne CJ, Payne AR, Gibson SC, et al. Is there still a role for preoperative 12-lead electrocardiography? World J Surg 2011;35:2611-6.

25. Wolters T, Weber M, Neumann T, et al. Pathologic $Q$ Waves and Prolonged QTc Time in Preoperative ECG Are Predictive for Perioperative Cardiovascular Events. World J Cardiovasc Dis 2014;04:498-509.

26. Morganroth J, Shah RR, Scott JW. Evaluation and management of cardiac safety using the electrocardiogram in oncology clinical trials: focus on cardiac repolarization (QTc interval). Clin Pharmacol Ther 2010;87:166-74.

27. Staikou C, Stamelos M, Stavroulakis E. Impact of anaesthetic drugs and adjuvants on ECG markers of torsadogenicity. Br J Anaesth 2014;112:217-30.

28. Yu SK, Tait G, Karkouti K, et al. The safety of perioperative esmolol: a systematic review and meta-analysis of randomized controlled trials. Anesth Analg 2011;112:267-81.

29. Landoni G, Turi S, Biondi-Zoccai G, et al. Esmolol reduces perioperative ischemia in noncardiac surgery: a meta-analysis of randomized controlled studies. J Cardiothorac Vasc Anesth 2010;24:219-29.

30. Figueredo E, Garcia-Fuentes EM. Assessment of the efficacy of esmolol on the haemodynamic changes induced by laryngoscopy and tracheal intubation: a meta-analysis. Acta Anaesthesiol Scand 2001;45:1011-22.

31. Poveda-Jaramillo R, Monaco F, Zangrillo A, et al. Ultra-ShortActing $\beta$-Blockers (Esmolol and Landiolol) in the Perioperative Period and in Critically III Patients. J Cardiothorac Vasc Anesth 2018;32:1415-25. 